\title{
$\left.{ }_{\text {SCIENCE }}^{\text {MATRIX }}\right\}$ Mascot Search Results
}

\section{Protein View}

Match to: PGTE_SALTY Score: 147 Expect: 2.5e-011

Outer membrane protease E OS=Salmonella typhimurium (strain LT2 / SGSC1412 / ATCC 700720) GN=pgtE PE=3 SV=2

Nominal mass $\left(\mathrm{M}_{\mathrm{r}}\right)$ : 35033; Calculated $\mathrm{pI}$ value: $\mathbf{5 . 2 8}$

NCBI BLAST search of PGTE SALTY against $n r$

Unformatted sequence string for pasting into other applications

Taxonomy: Salmonella enterica subsp. enterica serovar Typhimurium str. LT2

Variable modifications: Carbamidomethyl (C), Oxidation (M)

Cleavage by Trypsin: cuts C-term side of KR unless next residue is $P$

Number of mass values searched: 69

Number of mass values matched: 19

Sequence Coverage: 59\%

Matched peptides shown in Bold Red

1 MKKHAIAVMM IAVFSESVYA ESALFIPDVS PDSVTTSLSV GVLNGKSREI

51 VYDTDTGRKL SQLDWKIKNV ATLQGDLSWE PYSFMTLDAR GWTSLASGSG

101 HMVDHDWMSS EQPGWTDRSI HPDTSVNYAN EYDLNVKGWL LOGDNYKAGV

151 TAGYQETRFS WTARGGSYIY DNGRYIGNFP HGVRGIGYSQ RFEMPYIGLA

201 GDYRINDFEC NVLFKYSDWV NAHDNDEHYM RKLTFREKTE NSRYYGASID

251 AGYYITSNAK IFAEFAYSKY EEGKGGTQII DKTSGDTAYF GGDAAGIANN

301 NYTVTAGLOY RE

Show predicted peptides also

Sort Peptides By $\odot$ Residue Number $\bigcirc$ Increasing Mass $\bigcirc$ Decreasing Mass

$\begin{array}{rrrr}\text { Start - End } & \text { Observed } & \text { Mr (expt) } & \text { Mr (calc) } \\ 49-58 & 1168.5525 & 1167.5452 & 1167.5408 \\ 49-59 & 1296.6580 & 1295.6507 & 1295.6357 \\ 69-90 & 2514.2189 & 2513.2116 & 2513.1897 \\ 69-90 & 2530.1897 & 2529.1824 & 2529.1846 \\ 138-147 & 1193.6054 & 1192.5981 & 1192.5877 \\ 148-158 & 1152.5659 & 1151.5586 & 1151.5571 \\ 159-164 & 767.3754 & 766.3681 & 766.3762 \\ 165-174 & 1101.4869 & 1100.4796 & 1100.4887 \\ 175-184 & 1159.5862 & 1158.5790 & 1158.5934 \\ 185-191 & 780.3949 & 779.3876 & 779.3926 \\ 192-204 & 1531.7217 & 1530.7144 & 1530.7177 \\ 192-204 & 1547.7058 & 1546.6985 & 1546.7126 \\ 205-215 & 1398.6746 & 1397.6674 & 1397.6649 \\ 216-231 & 2051.8129 & 2050.8056 & 2050.8228 \\ 216-231 & 2067.8035 & 2066.7963 & 2066.8177 \\ 232-236 & 664.4184 & 663.4111 & 663.4068 \\ 244-260 & 1856.8642 & 1855.8569 & 1855.8628 \\ 261-269 & 1075.5406 & 1074.5333 & 1074.5386 \\ 283-311 & 2968.3641 & 2967.3568 & 2967.3635\end{array}$

\begin{tabular}{|c|c|c|}
\hline ppm & Miss & Sequence \\
\hline 4 & 0 & R.ELVYDTDTGR.K \\
\hline 12 & 1 & R.ELVYDTDTGRK.L \\
\hline 9 & 0 & K. NVATLQGDLSWEPYSFMTLDAR.G \\
\hline-1 & 0 & K.NVATLQGDLSWEPYSFMTLDAR.G Oxidation (M) \\
\hline 9 & 0 & K. GWLLQGDNYK . A \\
\hline 1 & 0 & K. AGVTAGYQETR.F \\
\hline-11 & 0 & R.FSWTAR.G \\
\hline-8 & 0 & R.GGSYIYDNGR.Y \\
\hline-12 & 0 & R.YIGNFPHGVR.G \\
\hline-6 & 0 & R.GIGYSQR.F \\
\hline-2 & 0 & R.FEMPYIGLAGDYR . I \\
\hline-9 & 0 & R.FEMPYIGLAGDYR.I Oxidation (M) \\
\hline 2 & 0 & R.INDFECNVLFK.Y Carbamidomethyl (C) \\
\hline-8 & 0 & K. YSDWVNAHDNDEHYMR . K \\
\hline-10 & 0 & K.YSDWVNAHDNDEHYMR.K Oxidation (M) \\
\hline 7 & 1 & R.KLTFR.E \\
\hline-3 & 0 & R.YYGASIDAGYYITSNAK. I \\
\hline-5 & 0 & K. IFAEFAYSK.Y \\
\hline-2 & 0 & . TSGDTAYFGGDAAGIANNNY? \\
\hline
\end{tabular}

No match to: 608.3377, 644.0112, 662.2948, 666.0136, 832.2928, 833.0584, 855.0329, 856.5275, 870.5537, 871.0027, 908.5037,

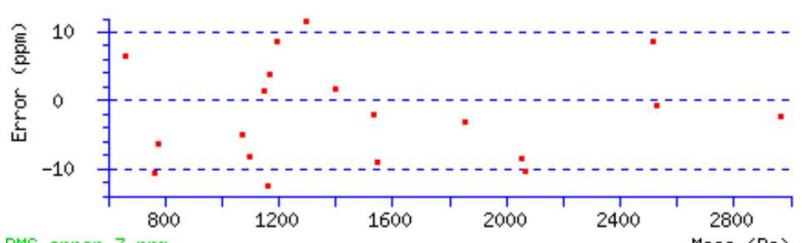

RHS error $7 \mathrm{mmm} \quad$ Mass (Da)

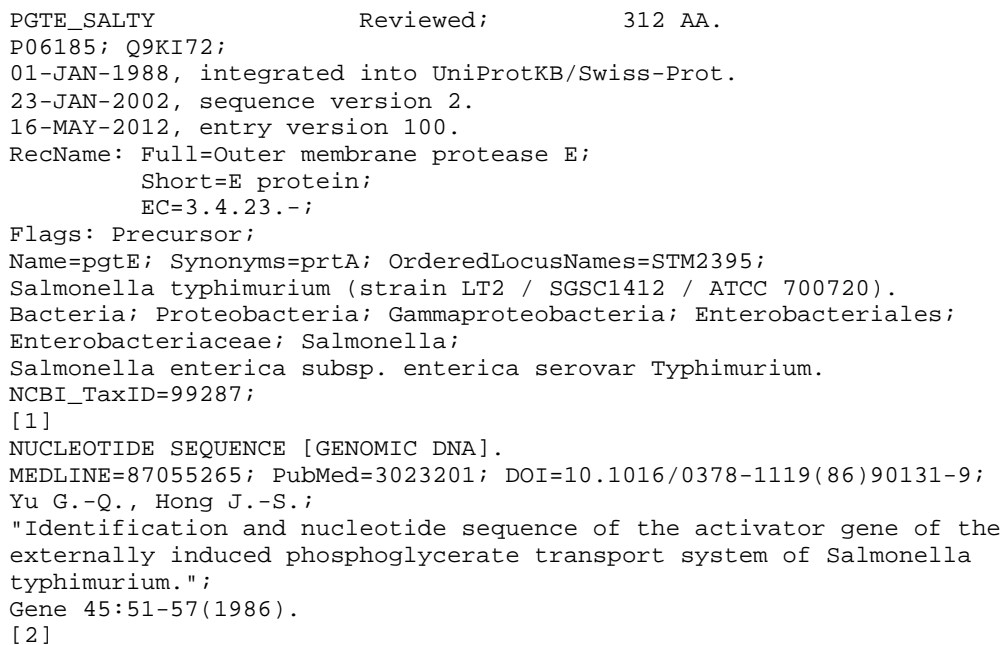




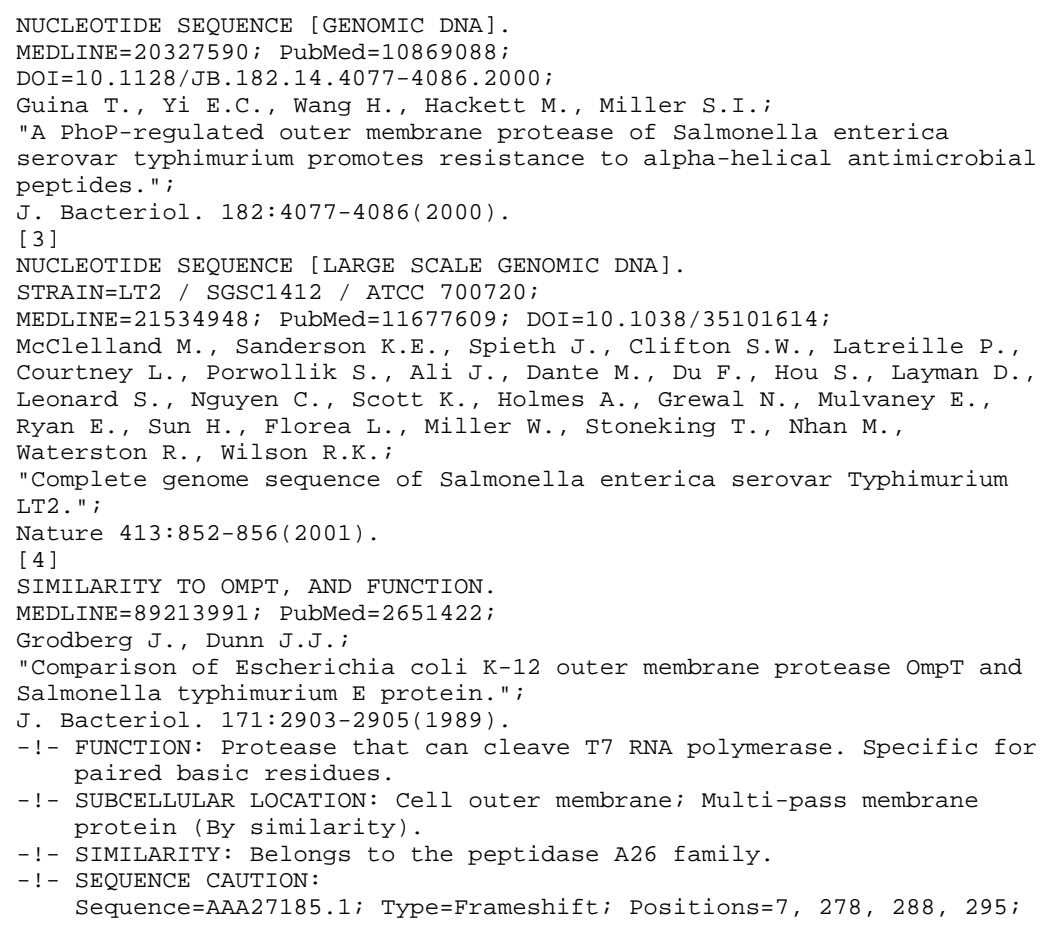

Copyrighted by the UniProt Consortium, see http://www.uniprot.org/terms Distributed under the Creative Commons Attribution-NoDerivs License

EMBL; M13923; AAA27185.1; ALT_FRAME; Genomic_DNA.

EMBL; AF239770; AAF85951.1; -; Genomic DNA.

EMBL; AE006468; AAL21295.1; -; Genomic_DNA.

$\mathrm{PIR} ; \mathrm{B} 28255 ; \mathrm{B} 28255$.

RefSeq; NP_461336.1; NC_003197.1.

ProteinModelPortal; P06185; -

MEROPS; A26.004; -

PRIDE; P06185; - .

GeneID; 1253917 ; -

GenomeReviews; AE006468_GR; STM2395.

KEGG; stm:STM2395; - .

PATRIC; 32383409; VBISalEnt20916_2533.

eggNOG; COG4571; -

HOGENOM; HOG000117799; - .

$\mathrm{KO}$; K08477; - .

OMA; SQLDWRI; - .

ProtClustDB; PRK10993; -

BioCyc; STYP99287:STM2395-MONOMER; -

GO; GO:0009279; C:cell outer membrane; IEA:UniProtKB-SubCell.

GO; GO:0016021; C:integral to membrane; IEA:UniProtKB-KW.

GO; GO:0005886; C:plasma membrane; IEA:UniProtKB-KW.

GO; GO:0004190; F:aspartic-type endopeptidase activity; IEA:UniProtKB-KW.

$\mathrm{GO} ; \mathrm{GO}: 0006508$; P:proteolysis; IEA:UniProtKB-KW.

Gene3D; G3DSA:2.40.128.90; Peptidase_A26; 1 .

InterPro; IPR020080; OM_adhesin/peptidase_omptin.

InterPro; IPR023619; Peptidase_A26.

InterPro; IPR020079; Peptidase_A26_CS.

InterPro; IPR000036; Peptidase_A26_omptin.

Pfam; PF01278; Omptin; 1 .

PIRSF; PIRSF001522; Peptidase_A26; 1.

PRINTS; PR00482; OMPTIN.

SUPFAM; SSF69917; OM_adhesin/protease_omptin; 1.

PROSITE; PS00834; OMPTIN_1; 1 .

PROSITE; PSO0835; OMPTIN_2; 1

3: Inferred from homology;

Aspartyl protease; Cell membrane; Cell outer membrane;

Complete proteome; Hydrolase; Membrane; Protease; Reference proteome;

Signal; Transmembrane; Transmembrane beta strand.

SIGNAL $\quad 1 \quad 37 \quad$ Potential.

CHAIN $\quad 38 \quad 312$ Outer membrane protease E.

FTId=PRO_0000025818.

ACT_SITE $104 \quad 104 \quad$ By similarity.

ACT_SITE $106 \quad 106 \quad$ By similarity.

ACT_SITE $226 \quad 226 \quad$ By similarity.

ACT_SITE $228 \quad 228 \quad$ By similarity.

CONFLICT $296 \quad 296 \quad$ G $\rightarrow S$ (in Ref. 1 ).

SEQUENCE 312 AA; $35055 \mathrm{MW}$; 5A6841732AF2E8C4 CRC64;

MKKHAIAVMM IAVFSESVYA ESALFIPDVS PDSVTTSLSV GVLNGKSREL VYDTDTGRKL

SOLDWKIKNV ATLOGDLSWE PYSFMTLDAR GWTSLASGSG HMVDHDWMSS EOPGWTDRSI

HPDTSVNYAN EYDLNVKGWL LQGDNYKAGV TAGYQETRFS WTARGGSYIY DNGRYIGNFP HGVRGIGYSQ RFEMPYIGLA GDYRINDFEC NVLFKYSDWV NAHDNDEHYM RKLTFREKTE NSRYYGASID AGYYITSNAK IFAEFAYSKY EEGKGGTQII DKTSGDTAYF GGDAAGIANN NYTVTAGLQY RF 